\title{
Recursive Graphical Construction of Tadpole-Free Feynman Diagrams and Their Weights in $\phi^{4}$-Theory
}

\author{
A. Pelster and K. Glaum \\ Institut für Theoretische Physik, Freie Universität Berlin, \\ Arnimallee 14, D-14195 Berlin, Germany \\ E-mails: pelster@physik.fu-berlin.de,glaum@physik.fu-berlin.de
}

\begin{abstract}
We review different approaches to the graphical generation of the tadpole-free Feynman diagrams of the self-energy and the one-particle irreducible four-point function. These are needed for calculating the critical exponents of the euclidean multicomponent scalar $\phi^{4}$-theory with renormalization techniques in $d=4-\epsilon$ dimensions.
\end{abstract}

\section{INTRODUCTION}

In 1982 Hagen Kleinert proposed a program for systematically constructing all Feynman diagrams of a field theory together with their proper weights by graphically solving a set of functional differential equations [1]. It relies on considering a Feynman diagram as a functional of its graphical elements, i.e., its lines and vertices. Functional derivatives with respect to these graphical elements are represented by removing lines or vertices of a Feynman diagram in all possible ways. With these graphical operations, the program proceeds in four steps. First, a nonlinear functional differential equation for the free energy is derived as a consequence of the field equations. Subsequently, this functional differential equation is converted to a recursion relation for the loop expansion coefficients of the free energy. From its graphical solution, the connected vacuum diagrams are constructed. Finally, all diagrams of $n$-point functions are obtained by removing lines or vertices from the connected vacuum diagrams. This program was recently used to systematically generate all Feynman diagrams of QED [2] and of $\phi^{4}$-theory in the disordered, symmetric phase [3] and the ordered, broken-symmetry phase [4, 5].

The present article reviews a modification of this program [6]. The aim is to construct directly the Feynman diagrams of $n$-point functions which are relevant for the renormalization of a field theory. To this end we consider the self-energy and the one-particle irreducible four-point function of the euclidean multicomponent scalar $\phi^{4}$-theory as functionals of the free correlation function. As such they obey a closed set of functional differential equations which can be turned into graphical recursion relations. These are solved order by order in the number of loops, producing all one-particle irreducible diagrams with their proper weights. A subsequent absorption of all tadpole corrections in the lines leads to modified graphical recursion relations for the tadpole-free one-particle irreducible diagrams which are needed for calculating the critical exponents with renormalization techniques in $d=4-\epsilon$ dimensions. Finally, we elucidate how our procedure is related to the method of higher functional Legendre transformations which was also investigated in Ref. [1].

\section{SCALAR $\phi^{4}$-THEORY}

Consider a self-interacting scalar field $\phi$ with $N$ components in $d$ euclidean dimensions whose thermal fluctuations are controlled by the energy functional

$$
E[\phi]=\frac{1}{2} \int_{12} G_{12}^{-1} \phi_{1} \phi_{2}+\frac{1}{4 !} \int_{1234} V_{1234} \phi_{1} \phi_{2} \phi_{3} \phi_{4} .
$$

In this short-hand notation, the spatial arguments and tensor indices of the field $\phi$, the bilocal kernel $G^{-1}$, and the quartic interaction $V$ are indicated by simple number indices, i.e.,

$$
1 \equiv\left\{x_{1}, \alpha_{1}\right\}, \quad \int_{1} \equiv \sum_{\alpha_{1}} \int d^{d} x_{1}, \phi_{1} \equiv \phi_{\alpha_{1}}\left(x_{1}\right), \quad G_{12}^{-1} \equiv G_{\alpha_{1}, \alpha_{2}}^{-1}\left(x_{1}, x_{2}\right), \quad V_{1234} \equiv V_{\alpha_{1}, \alpha_{2}, \alpha_{3}, \alpha_{4}}\left(x_{1}, x_{2}, x_{3}, x_{4}\right) .
$$

The kernel is a functional matrix $G^{-1}$, while the interaction $V$ is a functional tensor, both being symmetric in their respective indices. The energy functional (2.1) describes $d$-dimensional euclidean $\phi^{4}$-theories generically. These are models for a family of universality classes of continuous phase transitions, such as the $O(N)$-symmetric $\phi^{4}$-theory which serves to derive the critical phenomena in dilute polymer solutions $(N=0)$, Ising- and Heisenberg-like magnets $(N=1,3)$, and superfluids $(N=2)$. In all these cases, the energy functional (2.1) is specified by the bilocal kernel 


$$
G_{\alpha_{1}, \alpha_{2}}^{-1}\left(x_{1}, x_{2}\right)=\delta_{\alpha_{1}, \alpha_{2}}\left(-\partial_{x_{1}}^{2}+m^{2}\right) \delta\left(x_{1}-x_{2}\right),
$$

and by the quartic interaction

$$
V_{\alpha_{1}, \alpha_{2}, \alpha_{3}, \alpha_{4}}\left(x_{1}, x_{2}, x_{3}, x_{4}\right)=\frac{g}{3}\left\{\delta_{\alpha_{1}, \alpha_{2}} \delta_{\alpha_{3}, \alpha_{4}}+\delta_{\alpha_{1}, \alpha_{3}} \delta_{\alpha_{2}, \alpha_{4}}+\delta_{\alpha_{1}, \alpha_{4}} \delta_{\alpha_{2}, \alpha_{3}}\right\} \delta\left(x_{1}-x_{2}\right) \delta\left(x_{1}-x_{3}\right) \delta\left(x_{1}-x_{4}\right),
$$

where the bare mass $m^{2}$ is proportional to the temperature distance from the critical point, and $g$ denotes the bare coupling constant. In this article we leave the kernel $G^{-1}$ in the energy functional (2.1) completely general, except for the symmetry with respect to its indices, and insert the physical value (2.3) only at the end. By doing so, we consider all statistical quantities derived from (2.1) as functionals of the free correlation function $G$ which is the functional inverse of the kernel $G^{-1}$ :

$$
\int_{2} G_{12} G_{23}^{-1}=\delta_{13}
$$

This allows us to introduce functional derivatives with respect to $G$ whose basic rule reflects the symmetry of its indices:

$$
\frac{\delta G_{12}}{\delta G_{34}}=\frac{1}{2}\left\{\delta_{13} \delta_{42}+\delta_{14} \delta_{32}\right\} .
$$

Such functional derivatives are represented graphically by removing one line from a Feynman diagram in all possible ways [1 5 . Thereby each line in a Feynman diagram represents a free correlation function

$$
1-2 \equiv G_{12},
$$

and each vertex represents an integral over the interaction

$$
\rangle \equiv-\int_{1234} V_{1234}
$$

Thus the differentiation (2.6) is illustrated graphically as

$$
\frac{\delta}{\delta 3-4} 1-2=\frac{1}{2}\{1 \multimap 34-2+1 \multimap 43 \multimap 2\}
$$

where the elements of Feynman diagrams are extended by an open dot with two labeled line ends representing the delta function:

$$
{ }_{1 \multimap 2}=\delta_{12} .
$$

\section{CONNECTED VACUUM DIAGRAMS}

By using natural units in which the Boltzmann constant $k_{B}$ times the temperature $T$ equals unity, the partition function is determined as a functional integral over the Boltzmann weight $e^{-E[\phi]}$, i.e.

$$
Z=\int \mathcal{D} \phi e^{-E[\phi]},
$$

and may be evaluated perturbatively as a power series in the interaction $V$. ¿From this we obtain the negative free energy $W=\ln Z$ as an expansion

$$
W=\sum_{l=1}^{\infty} W^{(l)},
$$

where the coefficients $W^{(l)}$ for each loop order $l \geq 2$ may be displayed for as connected vacuum diagrams constructed from the lines (2.7) and the vertices (2.8). As has been elaborated in detail in Ref. [3, the connected vaccum 
diagrams contributing to $W^{(l)}$ follow together with their weights from a graphical recursion relation which can be written diagrammatically for $l \geq 2$ as

$$
\left.\left.W^{(l+1)}=\frac{1}{6 l} \frac{\delta^{2} W^{(l)}}{\delta 1-2 \delta 3-4} \begin{array}{l}
1 \\
3 \\
4
\end{array}\right\rangle+\frac{1}{2 l} \frac{\delta W^{(l)}}{\delta 1-2}{ }_{2}^{1} \succ 0+\frac{1}{6 l} \sum_{k=1}^{l-2} \frac{\delta W^{(l-k)}}{\delta 1-2}\right\rangle_{2}^{1} \succ_{4}^{3} \frac{\delta W^{(k+1)}}{\delta 3} .
$$

This is iterated starting from the two-loop contribution

$$
W^{(2)}=\frac{1}{8} \bigcirc
$$

The right-hand side of (3.3) contains three different graphical operations. The first two are linear and involve one or two line amputations of the previous perturbative order. The third operation is nonlinear and mixes two different one-line amputations of lower orders. ए]

The connected vacuum diagrams resulting from the graphical recursion relation (3.3) together with their weights are shown up to $l=5$ loops in Ref. [3]. There we observed that the nonlinear operation in (3.3) does not lead to topologically new diagrams. It only corrects the weights generated from the first two linear operations. Continuing the solution of the graphical recursion relation (3.3) to higher loops is an arduous task. We have therefore automatized the procedure in Ref. [3] by computer algebra with the help of a unique matrix notation for Feynman diagrams. The corresponding MATHEMATICA program and higher-order results up to $l=7$ are available on the internet [7].

\section{ONE-PARTICLE IRREDUCIBLE DIAGRAMS}

Once the connected vacuum diagrams are known, the diagrams of the fully-interacting two-point function

$$
\boldsymbol{G}_{12}=\frac{1}{Z} \int \mathcal{D} \phi \phi_{1} \phi_{2} e^{-E[\phi]}
$$

and the connected four-point function

$$
\boldsymbol{G}_{1234}^{\mathrm{c}}=\frac{1}{Z} \int \mathcal{D} \phi \phi_{1} \phi_{2} \phi_{3} \phi_{4} e^{-E[\phi]}-\boldsymbol{G}_{12} \boldsymbol{G}_{34}-\boldsymbol{G}_{13} \boldsymbol{G}_{24}-\boldsymbol{G}_{14} \boldsymbol{G}_{23}
$$

follow from removing one or two lines in all possible ways, respectively [3,7]:

$$
\begin{aligned}
\boldsymbol{G}_{12} & =2 \int_{34} G_{13} G_{24} \frac{\delta W}{\delta G_{34}}, \\
\boldsymbol{G}_{1234}^{\mathrm{c}} & =2 \int_{56} G_{35} G_{46} \frac{\delta \boldsymbol{G}_{12}}{\delta G_{56}}-\boldsymbol{G}_{13} \boldsymbol{G}_{24}-\boldsymbol{G}_{14} \boldsymbol{G}_{23},
\end{aligned}
$$

Note that removing a line from the diagrams of $\boldsymbol{G}_{12}$ according to the first term of (4.4) leads to disconnected diagrams which are canceled by the second and the third term to yield the connected diagrams contributing to $\boldsymbol{G}_{1234}^{\mathrm{c}}$. Dropping all diagrams of $\boldsymbol{G}_{12}$ and $\boldsymbol{G}_{1234}^{\mathrm{c}}$ which would fall into two pieces by removing a line, one obtains the one-particle irreducible diagrams of the self-energy

$$
\Sigma_{12}=G_{12}^{-1}-G_{12}^{-1}
$$

with $\boldsymbol{G}^{-1}$ being the functional inverse of $\boldsymbol{G}$

\footnotetext{
${ }^{1}$ Note that the first two operations in $(3.3)$ were already used in Ref. [8] as heuristic algorithms to generate all topological different connected vacuum diagrams of the $\phi^{4}$-theory up to $l=8$ loops with a computer program. Their corresponding weights were then determined by combinatorial means with a second computer program.
} 


$$
\int_{2} \boldsymbol{G}_{12} \boldsymbol{G}_{23}^{-1}=\delta_{13}
$$

and the one-particle irreducible four-point function

$$
\Gamma_{1234}=-\int_{5678} \boldsymbol{G}_{15}^{-1} \boldsymbol{G}_{26}^{-1} \boldsymbol{G}_{37}^{-1} \boldsymbol{G}_{48}^{-1} \boldsymbol{G}_{5678}^{\mathrm{c}}
$$

Along these lines all diagrams and their weights were constructed which are relevant for the five-loop renormalization of the $\phi^{4}$-theory in $d=4-\epsilon$ dimensions [8 10].

At this stage the question arises whether the one-particle irreducible diagrams can also be generated in a direct graphical way without any reference to the connected vacuum diagrams. This is indeed possible and will be elaborated in detail in Ref. [6]. Here we restrict ourselves to present some of the results. To this end we extend the elements of Feynman diagrams by a double straight line representing the fully-interacting two-point function

$$
1=2=G_{12},
$$

and by a 2- and 4-vertex with a big open dot representing the self-energy and the one-particle irreducible four-point function

$$
\overbrace{1}^{1-2}=\Sigma_{12},
$$

respectively. It turns out that the self-energy $\Sigma$ follows from an integral equation which reads graphically

$$
1-\mathrm{O}^{2}=\frac{1}{2} \mathrm{O}_{2}+\frac{1}{6} 1 \bigcirc-2 \text {. }
$$

Thereby the connected two-point function $\boldsymbol{G}$ is obtained from (4.5) according to the Dyson equation

$$
1=2=1 \square^{2}+1 \longrightarrow \mathrm{O}=2 \text {. }
$$

Solving the Eqs. (4.11) and (4.12) iteratively necessitates the knowledge of the one-particle irreducible four-point function $\Gamma$. Its diagrams could be determined from

$$
\begin{aligned}
\left.{ }_{1}^{2}\right)_{4}^{3}= & 2 \frac{\delta^{1-O}-2}{\delta 3-4}+23-\mathrm{O}-5 \frac{\delta^{1-\mathrm{O}-2}}{\delta 5-6} 6-\mathrm{O}-4 \\
& -23-\mathrm{O}-5 \frac{\delta^{1-\mathrm{O}-2}}{\delta 5-4}-2 \frac{\delta^{1-\mathrm{O}-2}}{\delta 3-6} 6-\mathrm{O}-4 .
\end{aligned}
$$

However, such a procedure would have one disadvantage: removing a line in the diagrams of the self-energy $\Sigma$ also leads to one-particle reducible diagrams which are later on cancelled by the third and the fourth term in (4.13). As the number of undesired one-particle reducible diagrams occuring at an intermediate step of the calculation increases with the perturbative order, this procedure is quite inefficient in determining the diagrams of the one-particle irreducible four-point function $\Gamma$. By inserting (4.11) in (4.13) it turns out that we can derive another equation for $\Gamma$ whose iterative solution only involves one-particle irreducible diagrams:

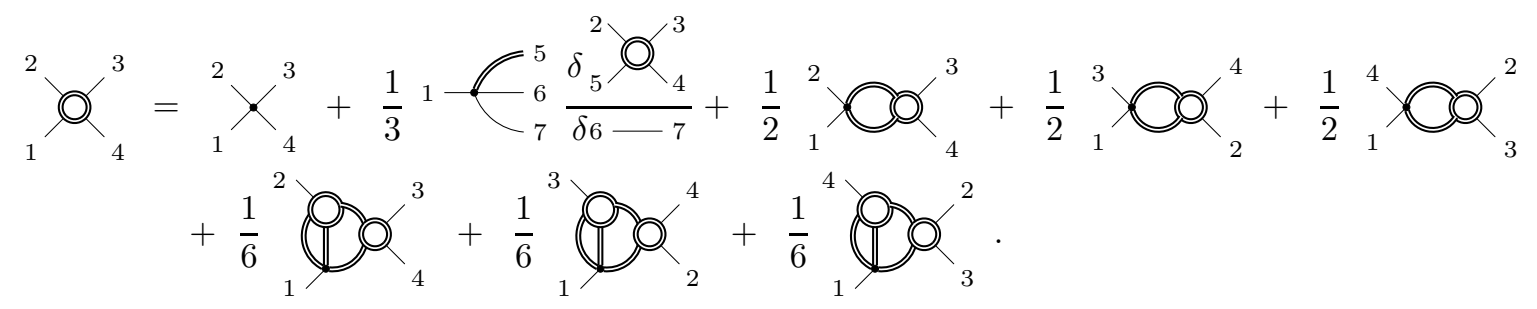

Note that Eqs. (4.11), (4.12) and (4.14) only generate one-particle irreducible diagrams for $\Sigma$ and $\Gamma$, if all lower loop orders contain one-particle irreducible diagrams. By induction this establishes that $\Sigma$ and $\Gamma$ only consist of one-particle irreducible diagrams. 


\section{TADPOLE-FREE ONE-PARTICLE IRREDUCIBLE DIAGRAMS}

In order to reduce the number of diagrams, we aim at substituting the free correlation function $G$ by a modified one $\tilde{\boldsymbol{G}}$. If we would fix $\tilde{\boldsymbol{G}}$ according to

$$
\tilde{\boldsymbol{G}}_{12}^{-1}=G_{12}^{-1}+\frac{1}{2} \int_{34} V_{1234} \tilde{\boldsymbol{G}}_{34},
$$

it would contain all repetitive one-loop corrections. This method was established in Ref. [1] to get rid of all oneparticle irreducible diagrams carrying tadpole corrections when calculating the $\beta$-function of the vacuum energy density up to five loops. Here, however, we go one step further by demanding instead of (5.1)

$$
\tilde{\boldsymbol{G}}_{12}^{-1}=G_{12}^{-1}+\frac{1}{2} \int_{34} V_{1234} \boldsymbol{G}_{34}
$$

which amounts to absorbing all momentum-independent line corrections into the mass [12]. The tadpole corrections of the modified correlation function $\tilde{\boldsymbol{G}}$ arising from (5.2) were already treated perturbatively in Ref. 何. It was shown that they lead to additional diagrams which cancel order by order all diagrams of one-particle irreducible $n$-point functions carrying any kind of tadpole correction. In Ref. [6] we elaborate that these inefficient cancellations can be circumvented as the remaining tadpole-free one-particle irreducible diagrams directly follow from a closed set of functional differential equations. To this end we consider the modified self-energy

$$
\tilde{\Sigma}_{12}=\tilde{\boldsymbol{G}}_{12}^{-1}-\boldsymbol{G}_{12}^{-1}
$$

and the one-particle irreducible four-point function $\Gamma$ as functionals of the modified correlation function $\tilde{\boldsymbol{G}}$. Extending the elements of Feynman diagrams by a wiggly line representing the modified correlation function

$$
1 \sim 2=\tilde{\boldsymbol{G}}_{12}
$$

and by a 2-vertex with a wiggly dot representing the tadpole-free self-energy

$$
1 \sim\{3\} 2=\tilde{\Sigma}_{12}
$$

our results read as follows. The tadpole-free self-energy $\tilde{\Sigma}$ obeys the integral equation

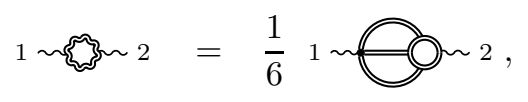

where the fully-interacting two-point function $G^{\mathrm{c}}$ is obtained from 5.3 according to the modified Dyson-equation

$$
1=2=1 \sim m 2+1 \sim\left\{\begin{array}{l}
=2 \\
=
\end{array}\right.
$$

Then the one-particle irreducible four-point function $\Gamma$ obeys

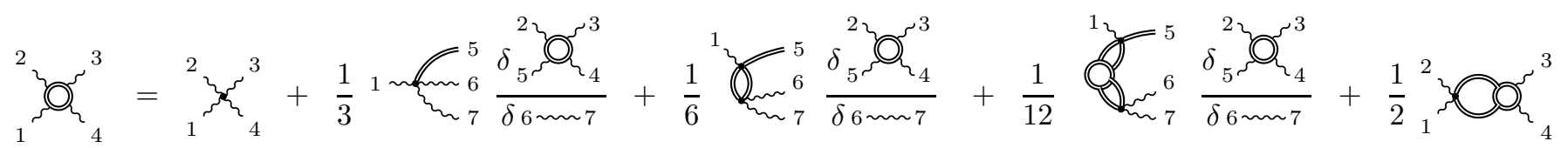

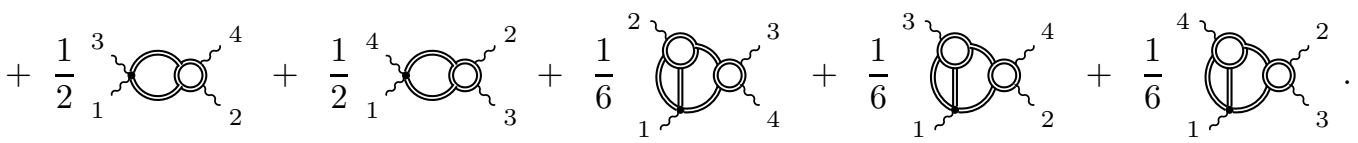

Iteratively solving Eqs. (5.6)-(5.8) in a graphical way leads to all tadpole-free diagrams of the self-energy and of the one-particle irreducible four-point function together with their weights. Up to five loops they are listed in Appendix A of Ref. [10]. Their respective quadratic and logarithmic divergencies in $d=4-\epsilon$ dimensions contribute to the $1 / \epsilon$-poles of the renormalization constants of the field $\phi$, the coupling constant $g$ and the mass $m^{2}$ within the minimal subtraction scheme, so that they determine the critical exponents of scalar $\phi^{4}$-theory. 


\section{ONE-PARTICLE IRREDUCIBLE DIAGRAMS WITHOUT LINE CORRECTIONS}

The number of diagrams can be even further reduced by substituting the free correlation function $G$ by the fullyinteracting two-point function $\boldsymbol{G}$ itself. Such a substitution was investigated in Ref. [1] within the framework of higher functional Legendre transformations. By considering the self-energy $\Sigma$ and the one-particle irreducible fourpoint function $\Gamma$ as functionals of the fully-interacting two-point function $\boldsymbol{G}$, it can be shown that they obey [1] 6 ]

$$
\begin{aligned}
& 1=\frac{1}{2}=2=\frac{1}{6}+2 \frac{\delta 1=\mathrm{O}=2}{\delta 3=4}+\underbrace{2}_{4} \frac{\delta 5=\mathrm{O}=6}{\delta 3=4} .
\end{aligned}
$$

Inserting (6.1) in (6.2) it turns out that the one-particle irreducible four-point function also follows from [6]

$$
\text { (1) }
$$

The graphical solution of the functional differential equations (6.1) and (6.2) or (6.3) leads to all one-particle irreducible diagrams which do not contain any line corrections. Once they are generated, we can recover the tadpole-free oneparticle irreducible diagrams according to the following algorithm [6]. At first we subtract the one-loop correction from the self-energy in order to obtain the tadpole-free self-energy

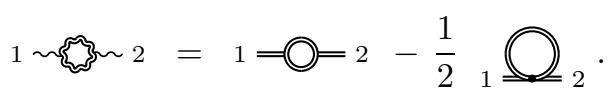

Iterating the modified Dyson equation (5.7), we then determine the tadpole-free diagrams of the fully-interacting twopoint function $\boldsymbol{G}$ where the lines represent the modified correlation function (5.4). Finally we insert this expansion into the one-particle irreducible diagrams of $\Sigma$ and $\Gamma$ determined from (6.1) and (6.2) or (6.3).

\section{SUMMARY}

We have reviewed different approaches to graphically generate tadpole-free one-particle irreducible diagrams together with their weights which are needed for calculating the critical exponents of $\phi^{4}$-theory. One approach is based on the graphical solution of Eqs. (5.6)-(5.8), the latter being more complex. The alternative approach consists of the simpler Eqs. (6.1) and (6.2) at the expense of subsequent iterations of the modified Dyson equation (5.7) with (6.4). In order to decide which of those approaches is more efficient, it is necessary to perform further analytic studies or to automatize them by computer algebra.

\section{ACKNOWLEDGMENTS}

A.P. is deeply indebted to Professor Dr. Hagen Kleinert for the opportunity to work with him as a scientific assistant. His universal physical knowledge, his many brilliant ideas and his truly encouraging personality cause a thrilling scientific environment in his research group. Both of us wish him a happy 60th birthday. Finally we thank Michael Bachmann, Dr. Boris Kastening, and Dr. Bruno van den Bossche for sharing our interest in the recursive graphical construction of Feynman diagrams. 
[1] H. Kleinert, Fortschr. Phys. 30, 187 and 351 (1982).

[2] M. Bachmann, H. Kleinert, and A. Pelster, Phys. Rev. D 61, 085017 (2000).

[3] H. Kleinert, A. Pelster, B. Kastening, and M. Bachmann, Phys. Rev. E 62, 1537 (2000).

[4] B. Kastening, Phys. Rev. E 61, 3501 (2000).

[5] A. Pelster and H. Kleinert, eprint: hep-th/0006153.

[6] A. Pelster, H. Kleinert, and K. Glaum, preprint.

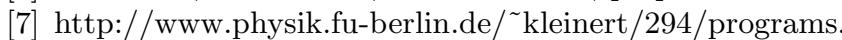

[8] J. Neu, MS Thesis (in German), FU-Berlin (1990).

[9] H. Kleinert, J. Neu, V. Schulte-Frohlinde, K.G. Chetyrkin, and S.A. Larin, Phys. Lett. B 272, 39 (1991); Phys. Lett. B 319, 545(E) (1993).

[10] H. Kleinert and V. Schulte-Frohlinde, Critical Properties of $\phi^{4}$-Theories (World Scientific, Singapore, 2001).

[11] B. Kastening, Phys. Rev. D 54, 3965 (1996); Phys. Rev. D 57, 3567 (1998).

[12] J. Küster and G. Münster, Z. Phys. C 73, 551 (1997). 